\title{
Ultrafast phase transitions after femtosecond laser irradiation of indium phosphide
}

\author{
J. Bonse, ${ }^{\text {a) }}$ S. M. Wiggins, and J. Solis \\ Instituto de Optica, CSIC, Serrano 121, 28006 Madrid, Spain
}

(Received 1 December 2003; accepted 2 June 2004)

\begin{abstract}
The structural transformation dynamics of single-crystalline indium phosphide irradiated with $150 \mathrm{fs}$ laser pulses at $800 \mathrm{~nm}$ has been investigated by means of time-resolved reflectivity measurements covering a time window from $150 \mathrm{fs}$ up to $500 \mathrm{~ns}$. The results obtained show that for fluences above the threshold of $0.16 \mathrm{~J} / \mathrm{cm}^{2}$ thermal melting of the material occurs on the time scale of 1-2 ps, while ablation at higher fluences $\left(>0.23 \mathrm{~J} / \mathrm{cm}^{2}\right)$ is preceeded by an ultrafast phase transition ("nonthermal melting") occurring within $400 \mathrm{fs}$ after the arrival of the pulse to the surface. The evolution of the reflectivity on a longer time scale shows a resolidification time typically around $25 \mathrm{~ns}$ after which an amorphous layer several tens of nanometer thick is formed on the surface.

(C) 2004 American Institute of Physics. [DOI: 10.1063/1.1776311]
\end{abstract}

\section{INTRODUCTION}

The dynamics of femtosecond laser-induced phase transitions has been intensively studied in semiconductors such as silicon ${ }^{1,2}$ and the III-V compounds gallium arsenide ${ }^{2,3}$ $(\mathrm{GaAs})$ and indium antimonide ${ }^{4}(\mathrm{InSb})$. Compared to these materials indium phosphide (InP), another III-V compound semiconductor, has a number of beneficial properties which make it interesting for high-frequency optoelectronic applications. ${ }^{5}$ Indeed, with the ever increasing intrinsic speed of photonic devices, InP has the potential to become the dominant material for use in future ultrahigh-speed detectors, optical switches, transistors, etc. ${ }^{6}$ However, only very few studies have investigated the dynamics of phase transitions of InP upon laser irradiation such that, to date, only nanosecond (ns)-laser pulse induced melting has been investigated by time-resolved optical measurements. ${ }^{7,8}$ Furthermore, these two studies do not provide a consistent interpretation of the transient evolution of the surface reflectivity upon melting.

With respect to the work on femtosecond (fs)-laser pulse irradiation, time-resolved measurements probing the carrier dynamics have been reported solely at carrier densities below any structural modification threshold ${ }^{9,10}$ while at higher fluences only static measurements of the final state of the material have been performed. ${ }^{11-13}$ In this paper we report a detailed study of the structural transformation dynamics of single-crystalline InP under fs-laser pulses, covering a time window from 150 fs up to $500 \mathrm{~ns}$. This has allowed us to investigate in detail melting, ablation, and rapid solidification phenomena in this material as well as nonthermal ultrafast phase transitions which occur at large fluences above the melting threshold. Additionally, our study includes the measurements of the reflectivity of liquid InP at two different wavelengths (514.5 and $800 \mathrm{~nm}$ ) and a quantitative estimation of the carrier density required for inducing an ultrafast nonthermal phase transition in InP.

\footnotetext{
${ }^{a)}$ Author to whom correspondence should be addressed; electronic mail: jbonse@io.cfmac.csic.es
}

\section{EXPERIMENTAL DETAILS}

The samples were $400 \mu \mathrm{m}$ thick polished (100) InP wafers (American Xtal Technology, Fremont, USA, $n$-doped). The laser used for irradiation was a chirped pulse amplification Ti:sapphire laser system (Spectra Physics, Spitfire), providing, at the sample, linearly polarized pulses of $\sim 150 \mathrm{fs}$ duration at a wavelength of $800 \mathrm{~nm}$. At this wavelength, in the linear absorption regime, the fundamental absorption mechanism in InP (band gap of $1.35 \mathrm{eV}$ ) is a direct interband transition.

The temporal evolution of the surface reflectivity upon irradiation has been measured using two different experimental setups with complementary time scales. A standard fs-resolution pump-probe (fs-PP) setup has been used to measure the reflectivity evolution for time delays between $150 \mathrm{fs}$ and $800 \mathrm{ps}$. For longer times (350 ps-500 ns) realtime reflectivity (RTR) measurements were performed simultaneously by means of a single sweep streak camera and a fast photodiode. In all cases the surface region was irradiated only once.

In the fs-PP experiment (see inset in Fig. 1), the laser pulse from the amplifier is first split into two orthogonally polarized pump and probe pulses. The pump pulse is then focused at normal incidence at the sample surface to a Gaussian spot diameter $\left(1 / e^{2}\right)$ of $\sim 100 \mu \mathrm{m}$. After passing through a delay line, the $p$-polarized probe pulse is focused at an angle of incidence of $12^{\circ}$ to a spot diameter of $\sim 18 \mu \mathrm{m}$ in the center of the region irradiated by the pump pulse. The reflected probe signal is then detected with a ns time-resolution photodiode coupled to a sampling oscilloscope. In the RTR measurements, a $1 \mu$ s long square pulse from a single-mode $\mathrm{Ar}^{+}$laser operating at $514.5 \mathrm{~nm}$ acts as the probe. The respective spot diameters for the pump and probe pulses in this case are $\sim 120 \mu \mathrm{m}$ and $\sim 30 \mu \mathrm{m}$. The reflected probe intensity is simultaneously recorded by a streak camera (time resolution of $350 \mathrm{ps}$ in a time window of $50 \mathrm{~ns}$ ) and by a photodiode/oscilloscope detection system (a few ns time resolution in a time window of $500 \mathrm{~ns}$ ). Further details regarding this RTR setup can be found elsewhere. ${ }^{14}$ 


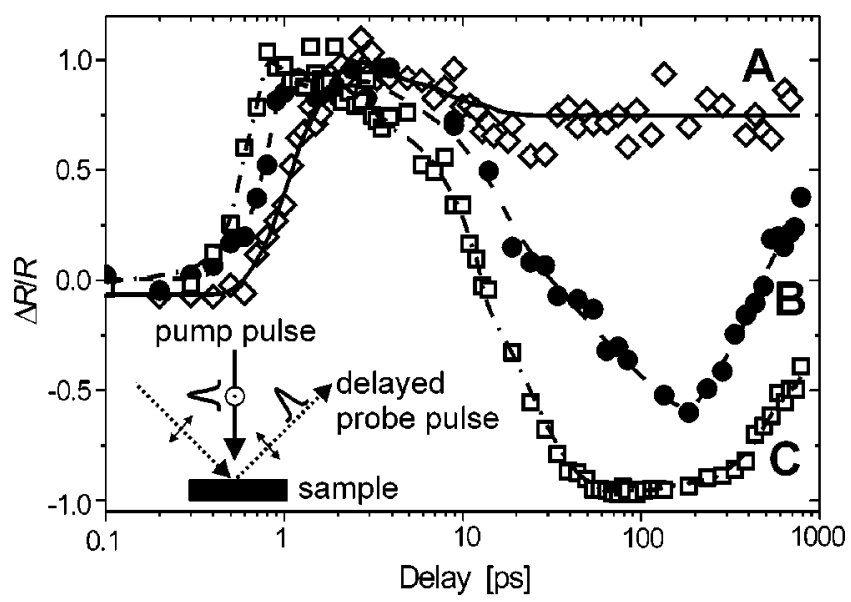

FIG. 1. Normalized surface reflectivity change as a function of time measured for three different pump fluence levels $\left(A: \phi_{0}=1.26 \phi_{\mathrm{m}}, B: \phi_{0}\right.$ $\left.=2.06 \phi_{\mathrm{m}}, C: \phi_{0}=5.35 \phi_{\mathrm{m}}\right)$. The lines are to guide the eye. The inset shows the experimental scheme of the fs pump-probe experiment.

\section{RESULTS AND DISCUSSION}

Figure 1 shows the temporal evolution of the reflectivity changes measured in the fs-PP experiment upon irradiation at three different pump peak fluences $\phi_{0}$ above the melting (curve $A$ ) and ablation thresholds (curves $B$ and $C$ ). In the curves, the reflectivity changes have been normalized to the value of the single-crystalline material $\left(R_{\mathrm{C}}[800 \mathrm{~nm}]=0.31\right)$ such that $\Delta R / R=\left\{R(t)-R_{\mathrm{C}}\right\} / R_{\mathrm{C}}$, where $R(t)$ corresponds to the measured reflectivity value at a given time delay. The measured melting and ablation thresholds are consistent within the experimental error with those given in Ref. 12 $\left(\phi_{\mathrm{m}}=0.16 \mathrm{~J} / \mathrm{cm}^{2}\right.$ and $\phi_{\mathrm{abl}}=0.23 \mathrm{~J} / \mathrm{cm}^{2}$, respectively). At a fluence level exceeding the melting threshold $\left(\phi_{0}=1.26 \phi_{\mathrm{m}}\right.$, curve $A$ ) but below the ablation threshold $\left(\phi_{\mathrm{abl}}=1.44 \phi_{\mathrm{m}}\right)$, a reflectivity change of more than $100 \%$ occurs within 1-2 ps. Subsequently, the reflectivity decreases slightly reaching finally a constant level of $\Delta R / R \sim 0.75$ at $\sim 18 \mathrm{ps}$. This behavior can be interpreted in terms of the formation of a thin liquid layer on the surface that propagates in depth, leading to the formation of an optically thick molten layer for times after $\sim 18 \mathrm{ps}$. The evolution of the reflectivity in such a scenario has been modeled by considering a thin film of molten material with variable thickness on top of a crystalline ( $c$ -InP) substrate. The optical model used takes into consideration the fully coherent superposition of all Fresnel reflections at all interfaces (air/liquid and liquid/solid) for the given wavelength $(800 \mathrm{~nm})$, angle of incidence $\left(12^{\circ}\right)$, and polarization ( $p$-polarized beam). ${ }^{15,16}$ Unfortunately, the refractive index $(n+i k)$ of liquid $\operatorname{InP}(l-\operatorname{InP})$ is not known and therefore as a reasonable approach the values for liquid GaAs (which has a band structure in the solid phase very similar to that of InP) have been used. The values used for simulation are thus $(n+i k)(l-\mathrm{GaAs})=(2.4+i 3.5)($ Ref. 3$)$ and $(n+i k)(c-\operatorname{InP})=(3.5+i 0.2)$ (Ref. 17) at the probing wavelength of $800 \mathrm{~nm}$. The simulations show a maximum $100 \%$ reflectivity increase for a molten film thickness of $\sim 50 \mathrm{~nm}$ followed by a saturation value corresponding to an optically thick layer with $\Delta R / R \sim 0.92$ for melt depths larger than $\sim 100 \mathrm{~nm}$. This confirms that the observed initial reflectivity increase up to a maximum of $\Delta R / R \sim 1.0$ (curve $A$ in Fig. 1) corresponds to a constructive interference effect during the propagation of the melt front into the material at a speed close to the longitudinal speed of sound in the solid $(\sim 5000 \mathrm{~m} / \mathrm{s}) .{ }^{18}$ The calculated saturation value of 0.92 is somewhat greater than that observed experimentally $(\Delta R / R$ $\sim 0.75$ ) which indicates that at this wavelength the approximation of using the optical properties of $l$-GaAs is not fully satisfactory.

At a fluence $\phi_{0}=2.06 \phi_{\mathrm{m}}$ (curve $B$ ), sufficient for ablation, a fast increase of the reflectivity can be observed within $450 \mathrm{fs}$ which is significantly shorter than the electronphonon relaxation time [ $\sim 2$ ps (Ref. 19)]. At the highest fluence $\left(\phi_{0}=5.35 \phi_{\mathrm{m}}\right.$, curve $\left.C\right)$, the reflectivity maximum is reached within $360 \mathrm{fs}$. Note that the maximum of curve $A$ is the same as for curves $B$ and $C$ (within the experimental error) although on a longer time scale. The subpicosecond reflectivity increase to a fluence-independent ${ }^{20}$ value has been observed before in other semiconductors such as silicon and gallium arsenide and interpreted in terms of a phase transition induced by electronic excitation. ${ }^{1-3,20}$ This phenomenon is usually referred to as nonthermal melting and is due to the creation of a high-density electron-hole plasma with carrier densities of the order of $10^{21}-10^{22} \mathrm{~cm}^{-3}$ that leads to a destabilization of the lattice structure before a thermal equilibrium with the carriers is achieved..$^{21,22}$ Both the time scale of the reflectivity increase $(<450 \mathrm{fs})$ in both curves $(B, C)$ and the initial fluence-independent value of the subpicosecond reflectivity maximum are consistent with the formation of a highly excited state of the material driven by electronic excitation. This process occurs homogeneously in the excited volume wherever the stability limit is exceeded. Since this volume is determined by the energy deposition depth of the pump pulse in the solid material [optical penetration depth $1 / \alpha \sim 300 \mathrm{~nm}$ in the present case], ${ }^{17}$ the stability limit can be reached simultaneously over a depth much larger than the optical penetration depth of the probe in the liquid $[1 / \alpha \sim 20 \mathrm{~nm}]]^{3}$ This explains the fluence independence of the initial reflectivity increase which is just a consequence of the formation of a disordered liquid state which can be formed within a few vibrational periods. ${ }^{20}$ All three criteria, i.e., (i) the reflectivity increase on a subpicosecond time scale, (ii) the fluence independence of this reflectivity level, and (iii) the observed fluence dependence of the time required to reach the high reflective state, clearly evidence the occurrence of nonthermal melting in InP upon fs-laser pulse irradiation.

In curve $B$, the subsequent decrease of the reflectivity to a minimum at $200 \mathrm{ps}$ is indicative of the onset of ablation. ${ }^{23}$ Beyond this point, the reflectivity rises again towards the reflectivity level of the liquid state. In curve $C$, the ensuing decrease in reflectivity is more pronounced and after $\sim 40 \mathrm{ps}$ the probe pulse is almost completely absorbed in the excited surface region. The reflectivity subsequently increases again after $\sim 300$ ps. The observation of a reflectivity minimum after a few hundred picoseconds is in excellent agreement with measurements of the onset of the ablation process in semiconductors and metals using fs time-resolved 
microscopy. ${ }^{23}$ In such studies the ablation is observed to be accompanied by the formation of a rarefraction wave inducing a transient Newton ring pattern.

Along with the formation of a rarefraction wave, additional contributions to the appearance of the reflectivity minimum might arise from evaporation/ejection of material from the surface as observed for ns pumping. ${ }^{8}$ This is supported by the fact that the equilibrium vapor pressure of phosphorus in InP increases rapidly above the melting point [ $\sim 27.5 \mathrm{~atm}$ at the melting temperature of $1335 \mathrm{~K}$ Ref. 24] which also explains why the fluence thresholds for melting and ablation are very close together. Whatever the actual mechanisms of material removal are, atomic force microscopy (AFM) investigations indicate that, at a fluence level of $3.6 \phi_{\mathrm{m}}=2.5 \phi_{\mathrm{abl}}$, a crater with a maximum depth of $\sim 80 \mathrm{~nm}$ is formed on the surface. ${ }^{12}$ The momentum transferred from the evaporating material to the excited surface layer, i.e., the recoil pressure, would also explain the protruding rim structure (height $\sim 20 \mathrm{~nm}$ and width $\sim 100-300 \mathrm{~nm}$ ) bordering the ablation crater as observed by AFM in Ref. 12.

In order to estimate quantitatively the characteristic carrier densities at the surface required for melting and ablation, an analysis of the crater depth reported in Ref. 12 at the fluence $3.6 \phi_{\mathrm{m}}$ can be used. By applying the formalism of Ref. 25 and knowing the crater depth $(80 \mathrm{~nm})$ and the linear optical constants of the crystalline phase, ${ }^{17}$ the two-photon absorption coefficient $\beta$ of $c$-InP has first been estimated to be $\sim 90 \mathrm{~cm} / \mathrm{GW}$ at the wavelength of $800 \mathrm{~nm}$. Hence, according to the theory presented in Ref. 26 , carrier density values of $2.0 \times 10^{22}$ and $3.2 \times 10^{22} \mathrm{~cm}^{-3}$ have been obtained for melting and ablation, respectively. At the ablation threshold, for example, where the laser intensity $I_{0}$ is $\sim 9$ $\times 10^{11} \mathrm{~W} / \mathrm{cm}^{2}$, both linear and two-photon absorption contribute significantly to the carrier generation in InP after fslaser pulse irradiation at $800 \mathrm{~nm}: \beta I_{0} \approx 8.1 \times 10^{4} \mathrm{~cm}^{-1}>\alpha$ $\approx 3.3 \times 10^{4} \mathrm{~cm}^{-1}$, where $\alpha$ and $\beta$ denote, respectively, the linear and the two-photon absorption coefficients of InP.

For the case of fs-laser-induced melting at fluences below the ablation threshold, RTR measurements have been performed to investigate the resolidification behavior on the ns time scale. Figure 2 shows the temporal evolution of the surface reflectivity (normalized to $R_{\mathrm{C}}[514.5 \mathrm{~nm}]=0.36$ ) for a fluence of $1.27 \phi_{\mathrm{m}}$ comparable to that of curve $A$ in Fig. 1. As shown in the streak camera transient of Fig. 2(a), the reflectivity rapidly increases to a level of $\Delta R / R \sim 0.56$ (leading to a value of $R_{\mathrm{L}}[514.5 \mathrm{~nm}]=0.56$ ). The thickness of the molten layer exceeds the optical penetration depth at $514.5 \mathrm{~nm}$ for a time duration of $\sim 15 \mathrm{~ns}$ and produces the observed reflectivity plateau. This change $(\Delta R / R \sim 0.56)$ at the plateau agrees well with the value of $\Delta R / R \sim 0.60 \mathrm{ob}-$ tained by optical simulations employing the refractive indices at $514.5 \mathrm{~nm}[(n+i k)(l-\mathrm{GaAs})=(1.5+i 2.7)($ Ref. 3) and $(n+i k)(c-\operatorname{InP})=(3.7+i 0.5) \quad($ Ref. 17) $)$. At this wavelength, the use of the optical constants of $l$-GaAs is a better approximation than in the case of $800 \mathrm{~nm}$. The difference between the optical properties of the liquid phases of InP and GaAs is most likely related to the different numbers of free carriers in the melt that arise from the respective ratios between ionic and covalent bondings.

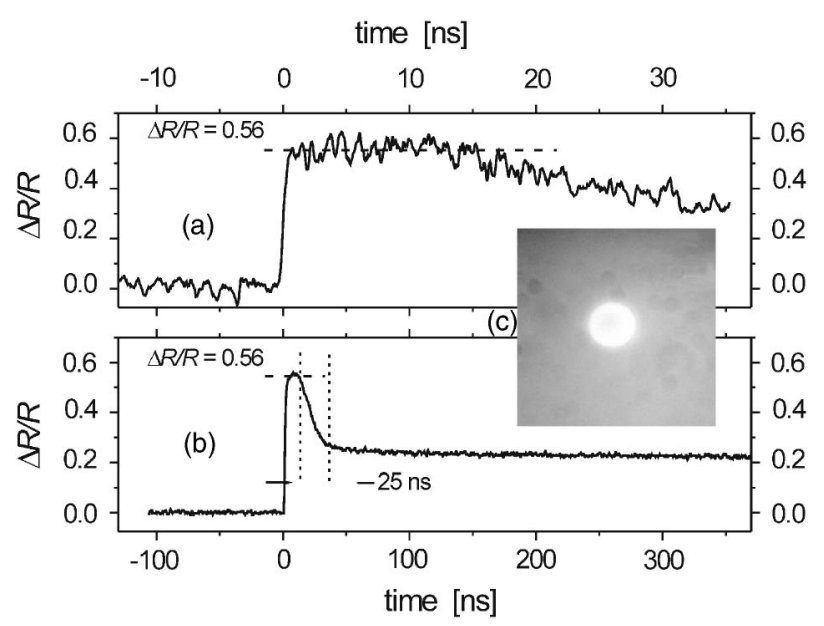

FIG. 2. Normalized surface reflectivity changes as a function of time for a fluence of $\phi_{0}=1.27 \phi_{\mathrm{m}}$ as measured with (a) streak camera and (b) photodiode. The arrival of the pump pulse to the surface occurs at time $t=0$. Note different time scales in (a) and (b). The maximum change measured $(\Delta R / R \sim 0.56)$ has been indicated in both plots. The dashed vertical lines in (b) mark the resolidification time interval ( $\sim 25 \mathrm{~ns})$. The inset (c) is an optical micrograph over an area of $160 \times 160 \mu \mathrm{m}^{2}$ corresponding to the laser irradiated spot.

The smooth decrease in reflectivity observed immediately after the plateau [Fig. 2(a)] indicates the onset of resolidification over the optically probed depth. Simultaneous measurements of the reflectivity with the photodiode [Fig. 2(b)] indicate a resolidification time of the order of $25 \mathrm{~ns}$. The observed resolidification times of some tens of nanoseconds after fs-laser-induced melting are, as expected, similar to those observed for ns-laser pulse irradiation. ${ }^{7,8}$ At a time $\sim 50 \mathrm{~ns}$ after the fs-pulse irradiation, after solid phase cooling, the reflectivity reaches its final level of $\Delta R / R \sim 0.22$, corresponding to the appearance of a bright spot in the irradiated region as shown in the optical micrograph of Fig. 2(c). Optical simulations of the surface reflectivity $(514.5 \mathrm{~nm}$, $18^{\circ}, s$-polarized beam), modeling a thin amorphous surface layer $[(n+i k)(a-\operatorname{InP})=(3.9+i 1.2)($ Ref. 27) $]$ on top of a crystalline substrate, show that the final surface reflectivity change of $\Delta R / R \sim 0.22$ is due to the formation of an amorphous layer with a thickness of several tens of nanometers. This is in good agreement with previous micro-Raman spectroscopy investigations which showed the formation of amorphous material upon fs-laser irradiation above the melting threshold. ${ }^{12}$

\section{CONCLUSIONS}

In summary, the dynamics of the fs-laser-induced phase transitions in single-crystalline InP has been analyzed by reflectivity measurements over a time scale between $150 \mathrm{fs}$ and $500 \mathrm{~ns}$. At low fluences above $\phi_{\mathrm{m}}$, thermal melting of the surface occurs within 1-2 ps and the liquid layer resolidifies within $\sim 40$ ns forming an amorphous top layer with a thickness of several tens of nanometers. At higher fluences above the ablation threshold, an ultrafast phase transition has been observed to occur within $\sim 400 \mathrm{fs}$. The reflectivity of this phase is fluence independent and shows an absolute value of 
$R \sim 0.63$ at $800 \mathrm{~nm}$. An estimation of the carrier density required to induce this phase transition leads to a value of $\geqslant 3 \times 10^{22} \mathrm{~cm}^{-3}$.

\section{ACKNOWLEDGMENTS}

This work was partially supported by the EU in the frame of the TMR Project XPOSE (Ref. No. HPRN-CT2000-00160). S.M.W. acknowledges the funding in the frame of the same project. J.B. acknowledges the funding of the CSIC through a contract in the frame of the I3P programme (Grant No. I3P-PC2002), cofunded by the European Social Fund. The authors would like to thank Professor J.M. Wrobel (University of Kansas City) for his courtesy of providing the samples.

${ }^{1}$ C. V. Shank, R. Yen, and C. Hirlimann, Phys. Rev. Lett. 50, 454 (1983).

${ }^{2}$ K. Sokolowski-Tinten, J. Bialkowski, and D. von der Linde, Phys. Rev. B 51, 14186 (1995).

${ }^{3}$ A. M.-T. Kim, J. P. Callan, C. A. D. Roeser, and E. Mazur, Phys. Rev. B 66, 245203 (2002)

${ }^{4}$ I. L. Shumay and U. Höfer, Phys. Rev. B 53, 15878 (1996).

${ }^{5}$ Properties, Processing and Applications of Indium Phosphide, edited by $\mathrm{T}$. P. Pearsall (IEE Publishing, London, 2000).

${ }^{6}$ S. Chandrasekhar, Solid-State Electron. 41, 1413 (1997).

${ }^{7}$ M. Yu. Aver'yanova, S. Yu. Karpov, Yu. V. Koval'chuk, V. E. Myachin,

Yu. V. Pogorel'skii, V. Z. Pyataev, and I. A. Sokolov, Sov. Tech. Phys. Lett. 12, 462 (1986).

${ }^{8}$ P. K. Kashkarov, V. Yu. Timoshenko, N. G. Chechenin, and A. N
Obraztsov, Laser Phys. 2, 790 (1992).

${ }^{9}$ U. Hohenester, P. Supancic, P. Kocevar, X. P. Zhou, W. Kütt, and H. Kurz, Phys. Rev. B 47, 13233 (1993).

${ }^{10}$ S. Nakashima, K. Mizoguchi, H. Harima, and K. Sakai, J. Lumin. 76, 6 (1998).

${ }^{11}$ J. Bonse, J. M. Wrobel, J. Krüger, and W. Kautek, Appl. Phys. A: Mater. Sci. Process. 72, 89 (2001)

${ }^{12}$ J. Bonse, J. M. Wrobel, K.-W. Brzezinka, N. Esser, and W. Kautek, Appl. Surf. Sci. 202, 272 (2002).

${ }^{13}$ A. Borowiec, M. Mackenzie, G. C. Weatherly, and H. K. Haugen, Appl. Phys. A: Mater. Sci. Process. 77, 411 (2003).

${ }^{14}$ J. Solis, J. Siegel, and C. N. Afonso, Rev. Sci. Instrum. 71, 1595 (2000).

${ }^{15}$ F. J. Pedrotti and L. S. Pedrotti, Introduction to Optics, 2nd ed. (PrenticeHall, Englewood Cliffs, NJ, 1993).

${ }^{16}$ For details of the model used see the appendix in J. Bonse, K.-W. Brzezinka, and A. J. Meixner, Appl. Surf. Sci. 221, 215 (2004).

${ }^{17}$ O. J. Glembocki and H. Piller, in Handbook of Optical Constants of Sol$i d s$, edited by E. D. Palik (Academic, Orlando, 1998).

${ }^{18}$ A. Dargys and J. Kundrotas, Handbook on Physical Properties of Ge, Si, GaAs and InP (Science and Encyclopedia, Vilnius, 1994).

${ }^{19}$ E. D. Grann, K. T. Tsen, and D. K. Ferry, Phys. Rev. B 53, 9847 (1996).

${ }^{20}$ See Fig. 2 in K. Sokolowski-Tinten, J. Bialkowski, M. Boing, A. Cavalleri, and D. von der Linde, Phys. Rev. B 58, R11805 (1998).

${ }^{21}$ P. Stampfli and K. H. Bennemann, Phys. Rev. B 42, 7163 (1990).

${ }^{22}$ P. Stampfli and K. H. Bennemann, Phys. Rev. B 49, 7299 (1994).

${ }^{23}$ D. von der Linde and K. Sokolowski-Tinten, Appl. Surf. Sci. 154-155, 1 (2000).

${ }^{24}$ K. J. Bachmann and E. Buehler, J. Electrochem. Soc. 121, 835 (1974).

${ }^{25}$ A. Rousse et al., Nature (London) 410, 65 (2001).

${ }^{26} \mathrm{~K}$. Sokolowski-Tinten and D. von der Linde, Phys. Rev. B 61, 2643 (2000).

${ }^{27}$ J. Stuke and G. Zimmerer, Phys. Status Solidi B 49, 513 (1972). 